Article

\title{
Prediction of global corrosion and corrosion products behavior in the primary circuit of LBE fast reactor
}

\author{
Jiewei $\mathrm{WU}^{1, \overline{\mathbf{T}}}$, Rongjun $\mathrm{WU}^{2, \overline{\boldsymbol{T}}}$, Yuqing WANG ${ }^{1}$, Jianbo $\mathrm{HE}^{1}$, Chen $\mathrm{HU}^{3}{ }^{3}$, Xian ZENG ${ }^{3}$, Muyi NI ${ }^{1, *}$ \\ 1 Sino-French institute of nuclear engineering and technology, Sun Yat-sen University, Zhuhai, China \\ 2 The Wuhan Second Boat Institution, Wuhan, China \\ 3 China Nuclear Power Technology Research Institute Co.,Ltd., Shenzhen, China \\ $\bar{T}$ These authors contributed equally to this work and should be considered co-first authors \\ * Correspondence: nimuyi@mail.sysu.edu.cn
}

\begin{abstract}
For better understanding the corrosion and corrosion products behavior in the primary circuit of lead-bismuth eutectic (LBE) coolant reactor, the concentration distribution of soluble impurities and the transport of solid particles are investigated through finite-element method. An axisymmetric model of the primary circuit of LBE reactor was constructed to accelerate the calculation the thermal hydraulic filed of circuit. The saturation concentration of solute $\mathrm{Fe}, \mathrm{Cr}$ and $\mathrm{Ni}$ in LBE coolant are identified through the equilibrium of their oxides and $\mathrm{PbO}$. And the very different saturation concentrations of $\mathrm{Fe} / \mathrm{Cr} / \mathrm{Ni}$ in $\mathrm{LBE}$ will lead to significant element selective corrosion. The migration of solid oxides particles in the primary circuit is also investigated by the Euler-Lagrange tracing model. The simulation shows that driving force for the movement of particles $>100 \mu \mathrm{m}$ is buoyancy, which lets particles float on a free surface, while particles $<10 \mu \mathrm{m}$ tend to suspend in coolant. However, the behavior of particles also depends on the formation position, the particles formed above the core have the high possibility of re-entering in the core.
\end{abstract}

Keywords: LBE; Corrosion products; CFD simulation; Fast reactor

\section{Introduction}

Lead-bismuth eutectic (LBE) alloy is main candidate coolant for advanced fast reactor and high-energy neutron spallation target coolant because of its excellent thermal conductivity, chemical inertness, and good neutronic characteristics [1]. However, in the hightemperature environment, the LBE coolant has a strong corrosive effect on the structure materials [2]. In a non-isothermal circuit, the LBE will corrode the material in the hightemperature zone, and the impurities will precipitate in the low-temperature zone due to the reduced solubility of the impurities, forming corrosion products (CPs) [3]. The main compositions of the $\mathrm{CPs}$ are $\mathrm{PbO}, \mathrm{Fe}, \mathrm{Ni}, \mathrm{Cr}$, and their oxides [4]. According to previous studies, controlling the oxygen concentration in LBE is an effective method to reduce the corrosion on structure materials [5]. And for typical structural steel, microscopic experiments had revealed the corrosion mechanism in LBE $[6,7]$. Therefore, it is necessary to investigate the structure corrosion and $\mathrm{CPs}^{\prime}$ behavior in the primary circuit of reactor in order to provide important information for the design of impurity filtration and removal systems so as to ensure the long-term stable operation of the reactor and maintain the security of the reactor $[8,9]$.

In this article, basing on a normal LBE fast reactor design, the global corrosion on structure was investigated, the concentration distribution of impurity was modeled, and finally the migration behavior of corrosion products was predicted.

\section{Service condition of structure materials in LFR}




\subsection{Operation condition of typical LFR}

A single-phase 2-D asymmetrical model was constructed by a commercial finite-element-method (FEM) software. The total mesh number was about 70, 000 and the main bulk mesh size was $2.5 \mathrm{~cm}$ and there is a mesh refinement on the boundaries. The $\mathrm{y}+\mathrm{al}-$ gebraic turbulence model was used to simulate the turbulent flow within a RANS framework in that it is suitable for the internal flow and it supports the porous medium approximation.

The core is composed of large number of fuel rods and arranged parallelly in vertical and heat exchanger (HX) is of U-shape tubes with complex geometry. Thus, detailed simulation of core and HX will consume a lot of computing resources. To resolve this problem, a porous medium approximation was used to model core and HX to match the expected pressure drops, which had shown good reliability according ref. $[9,10]$.

To realize the porous medium approximation, porosity and the permeability of the core and HX were described as Eq.(1):

$$
\begin{aligned}
& \varepsilon=\frac{1-\mathrm{V}_{\mathrm{s}}}{\mathrm{V}_{\mathrm{t}}} \\
& \mathrm{K}=\frac{\mathrm{Q} \mu \mathrm{L}}{\Delta \mathrm{PA}}
\end{aligned}
$$

Where $\varepsilon$ is porosity (dimensionless, $0<\varepsilon<1$ ); $V \mathrm{~s}$ is volume of solid part; $V \mathrm{t}$ is total volume of material; $\mathrm{K}$ is permeability; $\mathrm{Q}$ is volume flux through porous material; $\mu$ is fluid dynamic viscosity; $L$ is length of the pressure drop; $\Delta P$ is pressure difference of the fluid passes caused by the porous material; $A$ is cross section of porous material.

Table 1. Operation parameters of current LBE reactor designs [10]

\begin{tabular}{lllll}
\hline Design Parameters & CLEAR-I & SVBR-100 & MYRRHA & CASE \\
\hline Country & China & Russia & Belgium & China \\
Thermal capacity (MWth) & 10 & 280 & 100 & 100 \\
System pressure (MPa) & 0.05 & 6.7 & 0.17 & 3 \\
inlet/outlet $\left({ }^{\circ} \mathrm{C}\right)$ & $260 / 390$ & $340 / 490$ & $270 / 410$ & $250 / 350$ \\
Primary circulation & Natural & Forced & Forced & Forced \\
Reactor vessel & & $4.53 / 6.92$ & $8.0 / 12$ & $5 / 7$ \\
Flow rate of LBE coolant $(\mathrm{kg} / \mathrm{s})$ & 529.5 & 11760 & 9400 & 529.5 \\
Core: $\mathrm{D} / \mathrm{H}(\mathrm{m})$ & & $1.64 / 0.9$ & $1.83 / 1.75$ & $1.4 / 1.75$ \\
LBE inventory (ton) & 700 & 18.4 & 4500 & 110 \\
Average coolant flow rate $(\mathrm{m} / \mathrm{s})$ & & & 0.55 & 0.27 \\
The high coolant flow rate $(\mathrm{m} / \mathrm{s})$ & & & 2.37 & 1.88 \\
\hline
\end{tabular}

The mainstream conceptual design in the world and their principal parameters are listed in the Table 1. And a reference case was utilized to provide a baseline model. According to the design of MYRRHA [10,11], the porosity of reactor core is set to 0.3 and the porosity of HX is set to 0.62 in this baseline model, where permeability means the resistance of fluid flowing through porous medium in three dimensions. And a lower permeability means a stronger resistance. The flow of coolant in the core is assumed heterogenous: horizontal flow is very weak refer to LFR operation experience and the permeability is set to a very small value as $1 \times 10^{-12} \mathrm{~m}^{2}$; The strong vertical flow makes the pressure drop of core equal to 1.7 bars.

Primary pump was simulated by applying volume force to the domain of the pump to match the total pressure drop of the primary circuit. The volume force of the pump was set to $1.2 \times 10^{6} \mathrm{~Pa} / \mathrm{m}$ and the height was $0.2 \mathrm{~m}$. However, it was found in the simulation result that the flow velocity through the pump was incorrect and divergency happened. To improve the model, another porous model was utilized to configurate the LBE flow in pump [9]. And to compensate the pressure drop, the vertical permeability of pump is set 
to $10^{-8} \mathrm{~m}^{2}$ and the volume force is set to $1.3 \times 10^{6} \mathrm{~Pa} / \mathrm{m}$ to assure that the flow field of matching the design requirements.

The thermal power of core is set to $100 \mathrm{MW}$. The temperature of the coolant of the secondary circuit in the heat exchanger is $593.15 \mathrm{~K}$ and the heat exchange coefficient of $\mathrm{HX}$ is set to $0.85 \times 10^{5} \mathrm{~W} /\left(\mathrm{m}^{3} \cdot \mathrm{K}\right)$ to maintain the thermal circuit in the reactor.

\subsection{Flow field and temperature field}
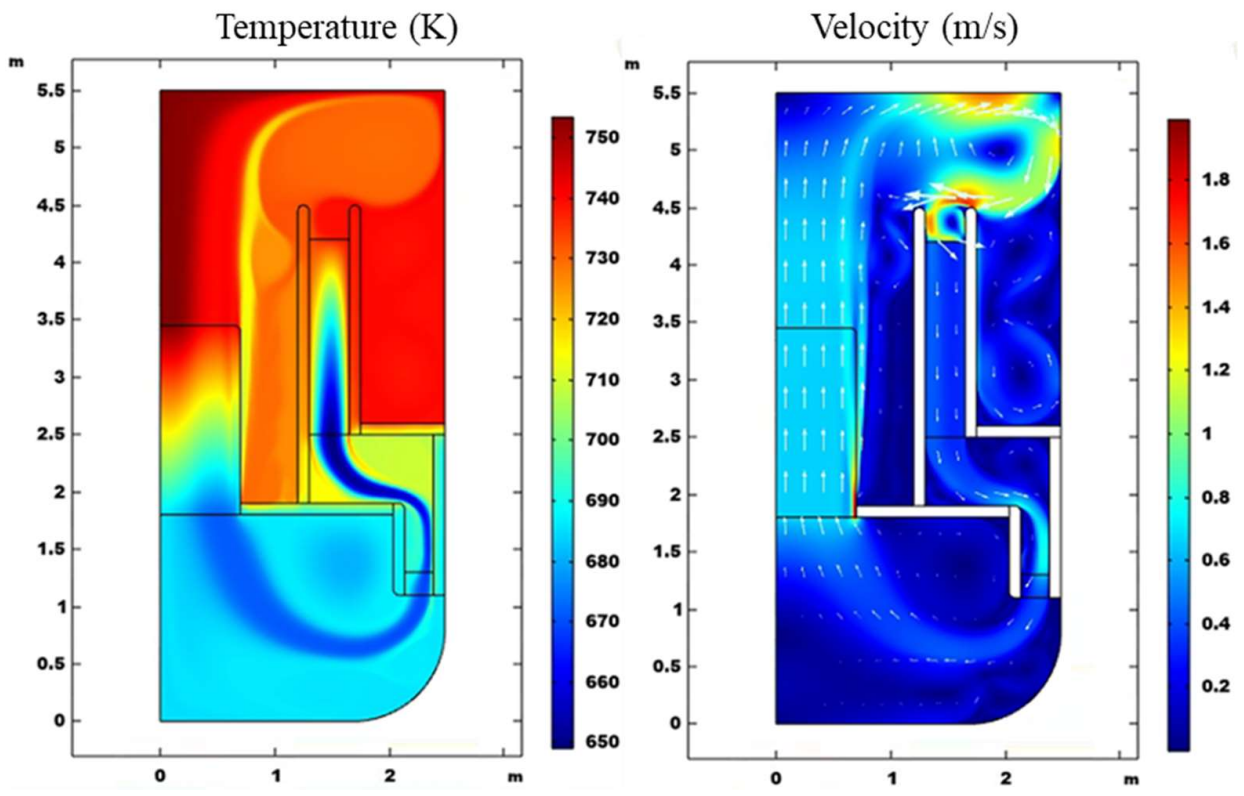

Figure 1. Flow field and temperature field of a typical LBE reactor section.

As shown in the Fig.1, the maximum temperature is out of the core as $753 \mathrm{~K}$ and the minimum temperature is in the $\mathrm{HX}$ as $650 \mathrm{~K}$. The temperature difference is around $67 \mathrm{~K}$. The maximum velocity in the circuit is $1.5 \mathrm{~m} / \mathrm{s}$ at inlet of the HX. We can also see that the horizontal flow velocity in the HX is almost 0 which means that the porous medium approximation can control very well the flow direction.

Meanwhile, the pressure of the primary circuit is calculated to verify whether porous medium approximation is set correctly. The reference value of pressure is set to 0 at the top of the reactor. The pressure distribution in the primary circuit and on the line $r=0.1$ $\mathrm{m}$ are shown in the Fig.2, and we can see that pressure decreases more quickly in the core and the porous medium causes another pressure drop of 1.7 bars. Consequently, desired pressure drops are gained through porous medium approximation. 


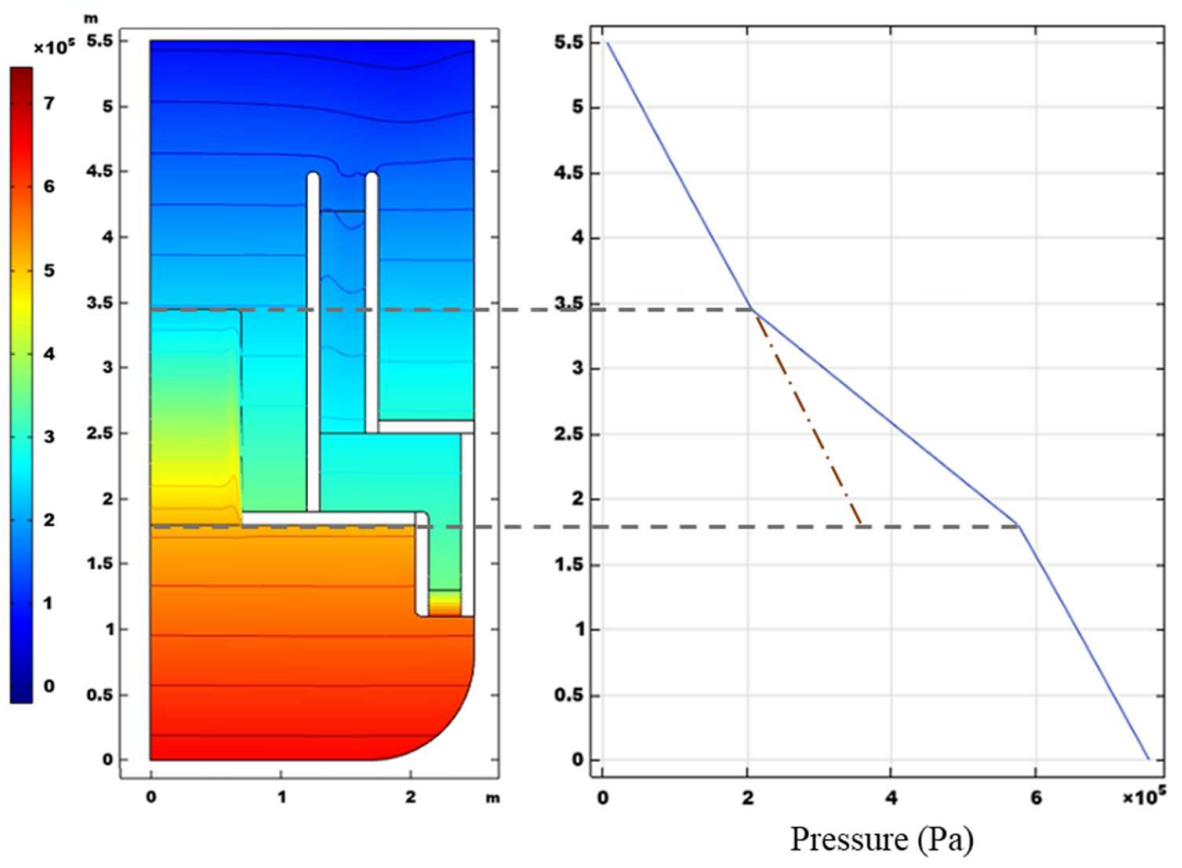

Figure 2. Relative pressure distribution in the LBE reactor and pressure drop on line $\mathrm{r}=0.1 \mathrm{~m}$.

\section{Corrosion products concentration distribution}

The structural material used in the LBE reactor is typical austenitic steel or martensitic steel [12]. Therefore, the main impurity dissolved into the LBE reactor will be Fe, $\mathrm{Cr}$ and Ni. The elements dissolved in the LBE will react with oxygen in the LBE. The reaction equations are shown in Eq.(2):

$$
\begin{aligned}
& 3 \mathrm{Fe}+4 \mathrm{O}=\mathrm{Fe}_{3} \mathrm{O}_{4} \\
& \mathrm{Ni}+\mathrm{O}=\mathrm{NiO} \\
& 2 \mathrm{Cr}+3 \mathrm{O}=\mathrm{Cr}_{2} \mathrm{O}_{3}
\end{aligned}
$$

In 2016, SCK-CEN has investigated the saturation concentration of Fe in LBE of different oxygen concentration [13] and the relation is given by Eq.(3)

$$
\log \left(c_{F e(L B E)}^{3} c_{O(L B E)}^{4}\right)=10.5-42935 / T
$$

However, because of the lack of $\mathrm{Cr}_{2} \mathrm{O}_{3}$ and $\mathrm{NiO}$ dissolution equilibrium constant, Zhang [1] developed a chemical kinetics model to predict the concentration of $\mathrm{Cr}$, Fe and $\mathrm{Ni}$ in different oxygen concentration. Based on the kinetics equilibrium of different oxides, the equations are shown in Eq.(4) Eq.(5) and Eq.(6).

$$
\begin{gathered}
C_{F e}=C_{F e, s} a_{p b}^{4 / 3}\left(\frac{C_{O, s}}{C_{O}}\right)^{4 / 3} \exp \left[\frac{1}{3 R T}\left(\Delta F_{\mathrm{Fe}_{3} O_{4}}-4 \Delta F_{P b O}\right)\right] \\
C_{C r}=C_{C r, s} a_{p b}^{3 / 2}\left(\frac{C_{O, s}}{C_{O}}\right)^{3 / 2} \exp \left[\frac{1}{2 R T}\left(\Delta F_{\mathrm{Cr}_{2} \mathrm{O}_{3}}-3 \Delta F_{P b 0}\right)\right]
\end{gathered}
$$




$$
C_{N i}=C_{N i, s} a_{P b}\left(\frac{C_{O, s}}{C_{O}}\right) \exp \left[\frac{1}{R T}\left(\Delta F_{N i O}-\Delta F_{P b O}\right)\right]
$$

Where $C_{s}$ is the saturation concentration in the oxygen-free environment, $a$ is the chemical activity in LBE and $\Delta F$ is the formation energy and they can all be find in the Handbook [14].

We checked the Fe concentration calculated by Eq.(3) and Eq.(4) under the same oxygen concentration and the result at $10^{-7}$ wt. $\%$ was shown in Fig.3. The kinetics models show good agreement with the experimental data and we can also use it to calculate the saturation concentration of $\mathrm{Cr}$ and $\mathrm{Ni}$.

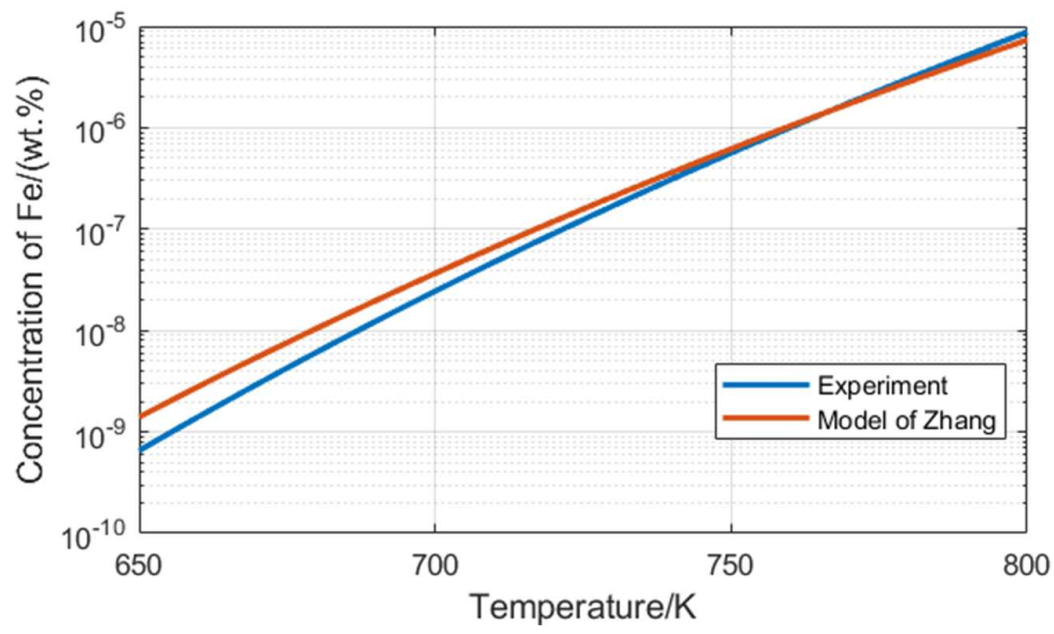

Figure 3. The concentration of Fe calculated by Eq.(3) (Experiment) and Eq.(4) (Zhang).

For LBE fast reactor, oxygen control system is a key subsystem to maintain the oxygen concentration within $10^{-9} \mathrm{wt} . \%$ to $10^{-5} \mathrm{wt} . \%$ to mitigate the structure corrosion [14]. In the baseline simulation, the oxygen concentration was hence set to $10^{-7} \mathrm{wt} . \%$. Since the oxygen concentration is too low to form $\mathrm{NiO}$, the equilibrium concentration of $\mathrm{Ni}$ was assumed to be like oxygen-free condition which is shown in Eq.(7).

$$
\log \left(\log \left(C_{\mathrm{Ni}_{s}}[w t . \%]\right)=1.53-843 / T\right.
$$

According to Eq.7, the saturation concentration of $\mathrm{Ni}$ is much greater than that of Fe. As a result, the dissolution of $\mathrm{Ni}$ is limited by the dissolution of Fe and we can assume that the concentration ratio of these 2 metals in the coolant is almost equal to the ratio of element concentration in the structure material. Typically, the Fe-Ni ratio in the steel is $70: 12[1]$ and accordingly, the Fe-Ni concentration ratio in the primary circuit is set to this value.

Coupled with thermal fluid analysis, the surface corrosion flux was established, the dynamic impurities concentration and distribution were calculated and shown in Fig.4. 


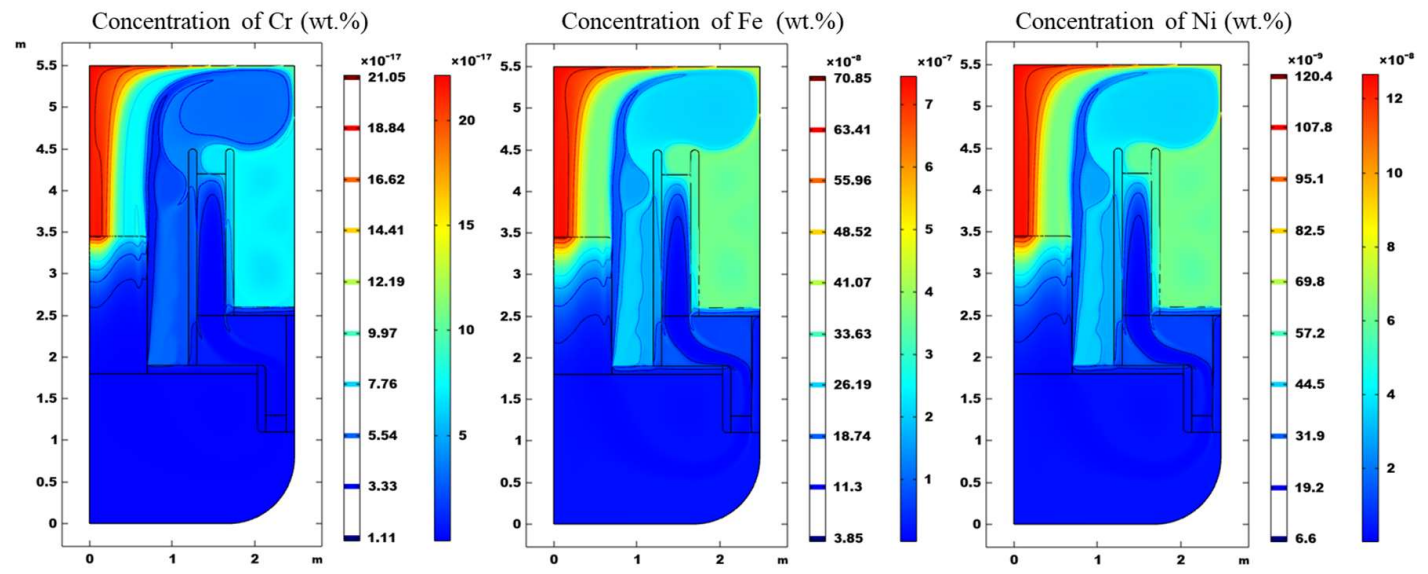

Figure 4. Concentration of $\mathrm{Cr}$, Fe and Ni in LBE primary circuit

The saturation of nickel in LBE is almost impossible to reach, unless a large amount of structural material were dissolved, which never been observed in former experiments. In the corrosion process, a layer of Fe-Cr spinel forms[15], which can restrain the concentration of $\mathrm{Ni}$ near the surface of the material, thereby reducing the dissolution of $\mathrm{Ni}$ from the structure material [16]. Consequently, in the conceptual design stage of the LBE fast reactor, the content of nickel in structure material should be restrained to prevent excessive dissolution and corrosion once the oxide film is destroyed.

\section{Tracing of CPs}

\subsection{Numerical model and basic assumptions}

According to previous researches, in the non-isothermal loop, particles directly formed in the reactor has the diameter from $0.01 \mu \mathrm{m}$ to $40 \mu \mathrm{m}$ and because of the agglomeration of elementary particles, the upper limit of particle diameter can even reach to 1 $\mathrm{mm}$ [17]. Thus, the particle diameter is set from $0.01 \mu \mathrm{m}$ to $1000 \mu \mathrm{m}$ in the following simulations. The density of the particle is set from $5200 \mathrm{~kg} / \mathrm{m}^{3}$ to $9530 \mathrm{~kg} / \mathrm{m}^{3}$ because the main compositions of oxide particle in $\mathrm{LBE}$ reactor are $\mathrm{Fe}_{3} \mathrm{O}_{4}$ and $\mathrm{PbO}$.

Under the normal operation conditions, the volume fraction of solid particles in the primary circuit is quiet low, which is about $10^{-6}$ [12]. The particles in LBE are dilute enough for a one-way coupling approach, i.e., the flow of coolant is not influenced by the movement of particles.

The equations describing the movement of particles is shown in Eq.(8):

$$
\frac{\partial\left(m_{p} v\right)}{\partial t}=F
$$

Where, $m_{p}$ is the mass of particle, $v$ is the velocity of particle and $F$ is the forces on particles.

The forces on particles are buoyancy, drag forces, virtual masses, pressure gradient and Brownian motion force [18]. The particles are assumed to be spherical and the drag force equation is shown in Eq.(9):

$$
\begin{aligned}
& F_{D}=\frac{1}{\tau_{p}} m_{p}(u-v) \\
& \tau_{p}=\frac{\rho_{p} d_{p}^{2}}{18 \mu}
\end{aligned}
$$

Where, $\tau_{p}$ is the response time of particles; $u$ is the velocity of fluid; $v$ is the velocity of particle; $\rho_{p}$ is the density of particle; $d_{p}$ is the particle diameter and $\mu$ is the viscosity of fluid. 
Because the particle size can be as low as $0.01 \mu \mathrm{m}$, the influence of the Brownian motion force must be considered and the formula is shown in Eq.(10)

$$
F_{b}=\varsigma \sqrt{\frac{12 \pi k_{B} \mu T r_{p}}{\Delta t}}
$$

Where $\Delta t$ is time-step that solver takes; $k_{B}$ is Boltzmann constant, $1.380649 \times 10^{-23}$ $\mathrm{J} / \mathrm{K}$; $\mathrm{T}$ is temperature of coolant; $r_{p}$ is radius of particle; $\zeta$ is Gaussian distribution with average 0 and standard deviation.

Particle collisions with walls were approximated as elastic which means the conservation of tangential momentum after the rebound. All interactions between particles are neglected because of the low concentration of particles. When the particles re-enter the core, they will be stopped by the sticky boundary condition in that the porous medium approximation is not accurate enough to predict the particle behavior in the core.

Due to the existence of turbulence and Brownian motion force, particles move constantly. There is thus no steady state solution for particle tracing. Besides, particle release number will influence the accuracy of the simulation results. Consequently, simulation time and release number should be determined before investigation of particle behavior in the primary circuit. The simulation is stopped when the system reaches to the pseudosteady state which means that the particles will vibrate around one place without going too far away.

A few particles are randomly released in the whole primary circuit except the core. The release number should be set large enough to obtain a statistically significant simulation result. The circuit is divided into 5 zones, core, HX and zone 1 to 3 shown in Fig. 5 and we count the particle number at the end time of simulation. When the particle number is greater than 500, the results vary slightly which means that 500 is large enough to predict the behavior of particles. The reason of not using 1000 is that it will cost lots of computing resources.

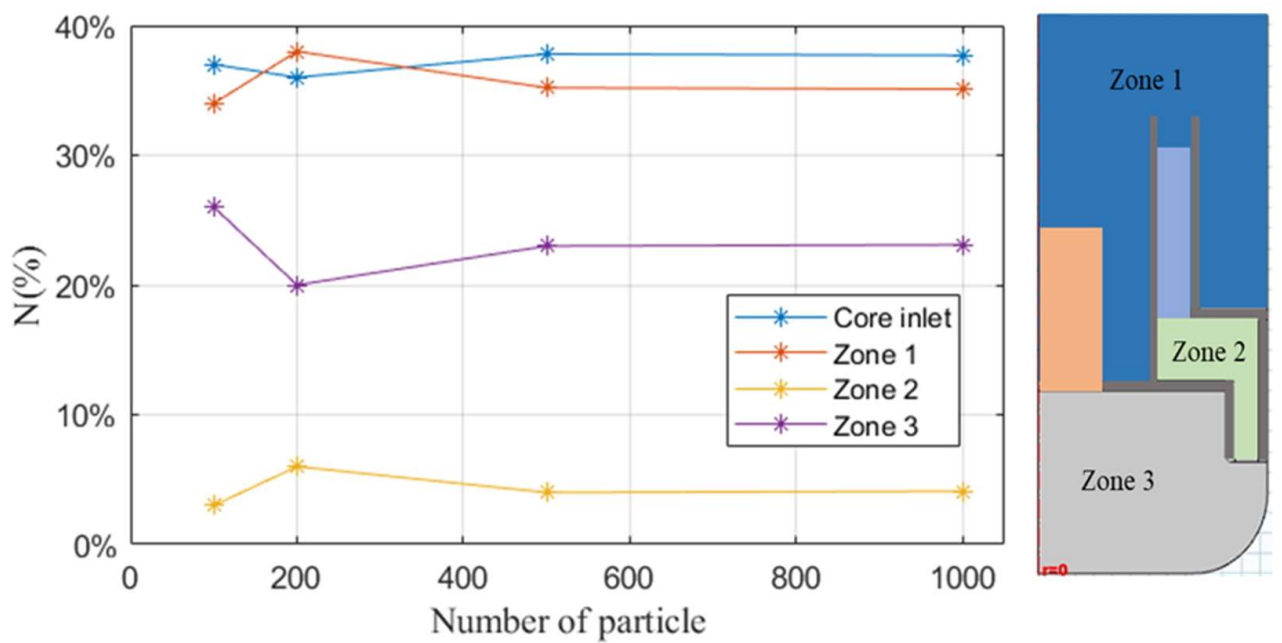
ber.

Figure 5. The relationship between the distribution of particle and the release num-

\subsection{Particles behavior in the primary circuit}

\subsubsection{Random particle release}

For the investigation of particle size influence, the oxides particles are assumed to be spherical and 8 diameter points are chosen to investigated which are $0.01 \mu \mathrm{m}, 0.1 \mu \mathrm{m}$, $1 \mu \mathrm{m}, 10 \mu \mathrm{m}, 40 \mu \mathrm{m}, 100 \mu \mathrm{m}, 500 \mu \mathrm{m}$ and $1000 \mu \mathrm{m}$. The density of particle is set to $5200 \mathrm{~kg} / \mathrm{m}^{3}$. The final distribution of particles of diameter $0.01 \mu \mathrm{m}, 40 \mu \mathrm{m}$ and $1000 \mu \mathrm{m}$ is shown in Fig.6. 

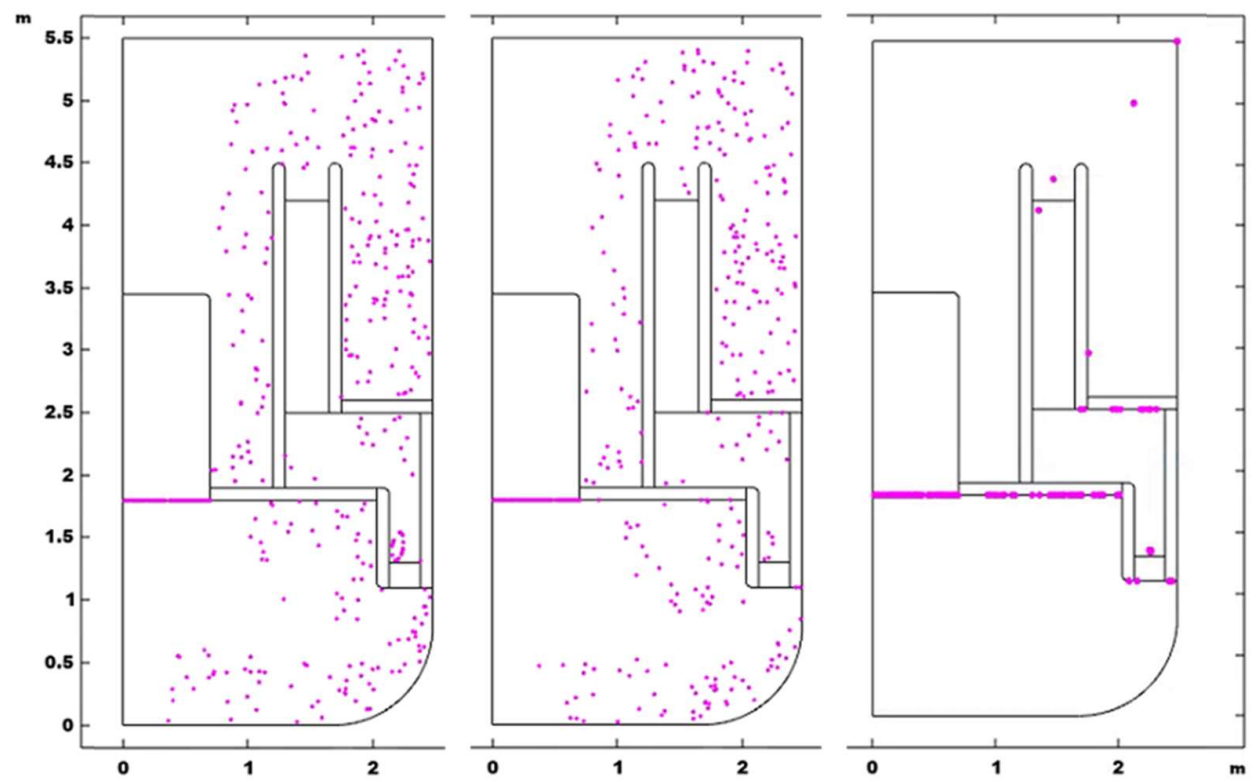

Figure 6. Final distribution of particles, $d_{p} 0.01 \mu \mathrm{m}$ (left), $d_{p} 40 \mu \mathrm{m}$ (middle), $d_{p} 1000$ $\mu \mathrm{m}$ (right).

It can be seen in Fig.6 that the final distributions with diameters of $0.01 \mu \mathrm{m}$ and 40 $\mu \mathrm{m}$ are slightly different, while the particles of $1000 \mu \mathrm{m}$ tend to float up due to the influence of buoyancy, and finally gather on the free surface or the interfaces between liquid and solid.

The particle distribution of different particle diameters is shown in Fig.7. The results are almost the same for the diameter smaller than $40 \mu \mathrm{m}$ which is correspondent to Fig.6. On the contrary, when particle diameter is greater than $100 \mu \mathrm{m}$, particles behave totally differently. More specifically, larger particles tend to float on free surface or liquid-solid interfaces because the buoyance becomes the dominating driving force for the particle movement. From Eq.(9), it can be found that while particle diameter increases, drag force decreases, the buoyance become decisive on particles behavior.

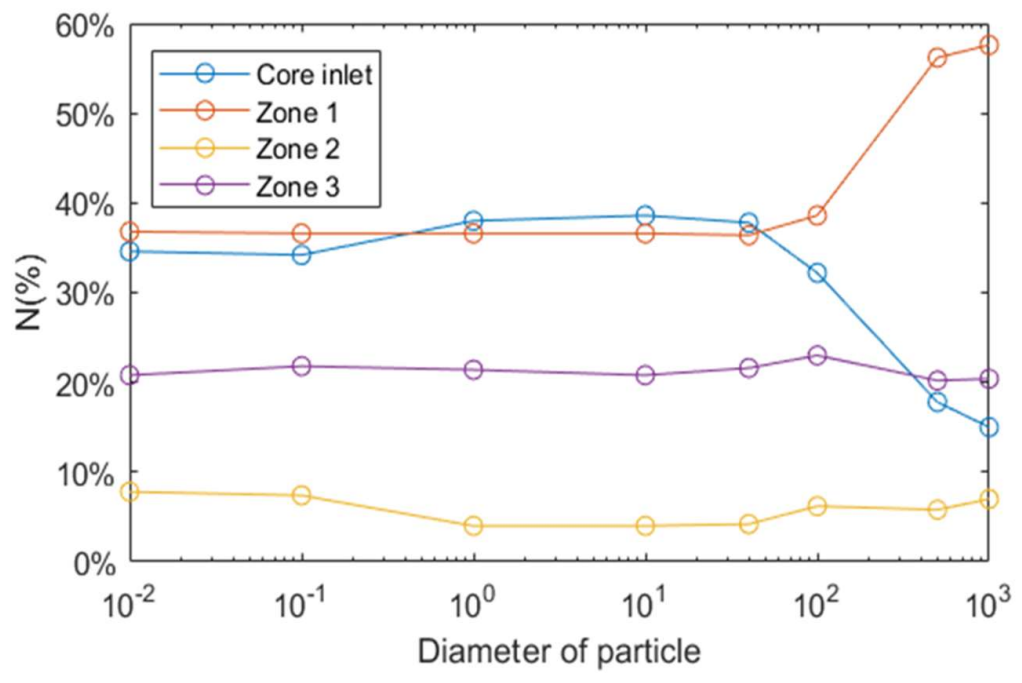

Figure 7. Distribution of particles of different diameter $(\mu \mathrm{m})$.

For the investigation of influence of particle density, 3 densities are chosen, 5200 $\mathrm{kg} / \mathrm{m}^{3}, 7400 \mathrm{~kg} / \mathrm{m}^{3}$ and $9530 \mathrm{~kg} / \mathrm{m}^{3}$. 


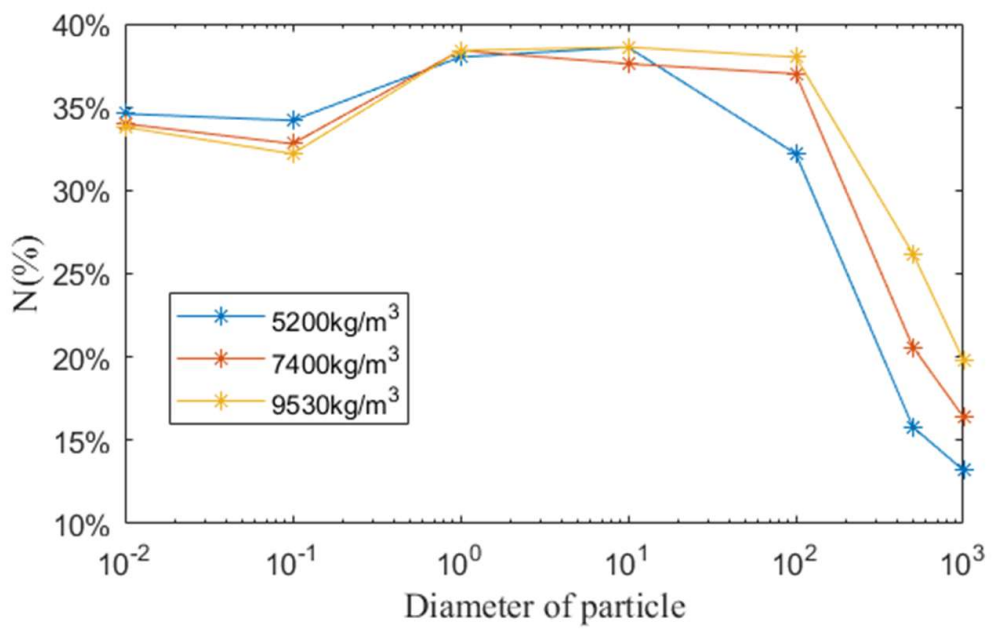

Figure 8 . The ratio of re-entering the core for particles of different diameter $(\mu \mathrm{m})$ and density.

As shown in Fig.8, when the particle diameter smaller than $100 \mu \mathrm{m}$, the ratio of reentering the core is not significantly related to the particle size. For the particle of diameter greater than $100 \mu \mathrm{m}$, the proportion of re-entering decreases significantly with the increase of particle density. As explained in the above section, the dominating driven force for the particle movement is buoyance and smaller density has greater buoyance leading to more particles floating on the free surface and less particles re-entering the core.

\subsubsection{The influence of initial position}

In the previous section, the particles released randomly from the core and HEx positions in the primary loop, while the formation position of the particle is not random in the LBE reactor. For example, above the core of a LBE reactor, impurity is more likely to be precipitated because of the large concentration difference. In order to explore the distribution of particles formed in different regions, the impact of different initial positions of particles with a diameter of $10 \mu \mathrm{m}$ on the final result was explored. Because when the particle diameter is greater than $100 \mu \mathrm{m}$, the buoyancy is greater, and finally all float on the free surface or the interface between liquid and structure material, which is of little research significance. Consequently, the particle with a particle diameter of $10 \mu \mathrm{m}$ is selected.

According to the previous section, the influence of density on the particle of diameter smaller than $100 \mu \mathrm{m}$ is not great, so the particles with density of $5200 \mathrm{~kg} / \mathrm{m}^{3}$ are taken as the research object. The initial position of the particles is divided into the following 6 regions. The regions are marked with different colors to distinguish them, and the particle distribution of the initial and final time are drawn, as shown in the Fig.9: 


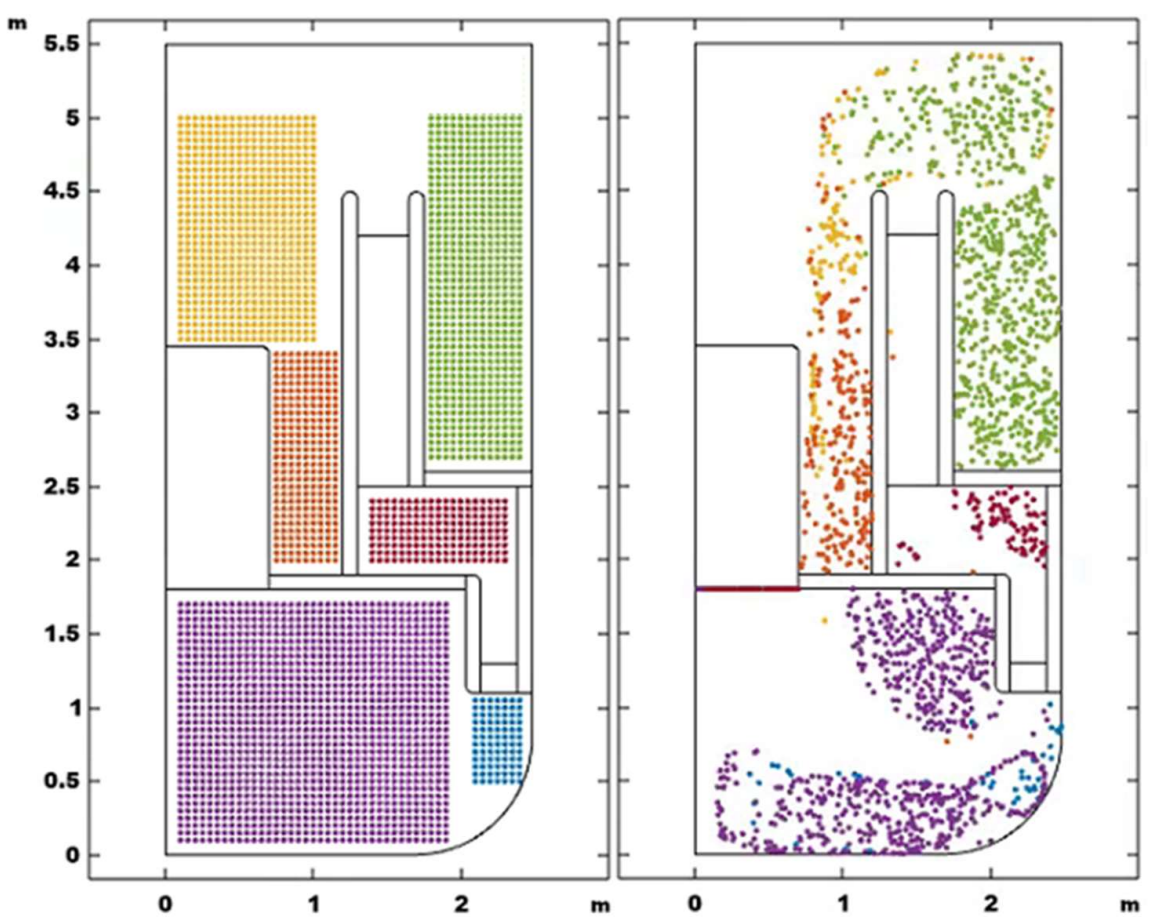

Figure 9. The final distribution of particles from different release positions

Fig.10 shows the proportion of the number of particles re-entering the core in different regions:

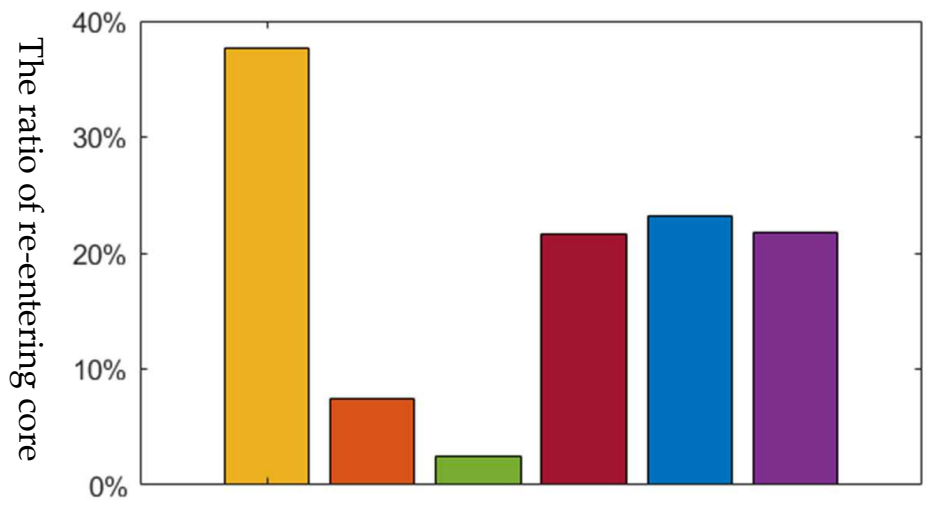

Figure 10. The ratio of particles re-entering core in different regions. The colors correspond to the colors of the particles in Fig.9

As shown in Fig.10, it can be obtained that the orange and green particles have a low probability of re-entering the core, while the particles above the core (yellow) have a higher probability of re-entering the core. We can see in Fig.1, there are lots of stagnant regions where orange and green particles are formed and for this reason, the particles are trapped by the eddies and they cannot re-enter the core. While for the particles formed above the core, they flow with the coolant and have greater possibility of re-entering the core.

\section{Conclusions}

Limited by the low accuracy of concentration measurement of metallic impurities in LBE, no experiments are now taken to study the concentration of impurities. At the same time, due to the lack of observation methods for small-size particles in LBE, the behavior of solid impurities also lacks experiments. Meanwhile, the migration behavior of metal 
impurities is very important in that the aggregation of large-diameter solid particles may cause directly blockage of the circuit, which may lead to severe accidents.

This article proposes preliminary ideas for the investigation of the behavior of impurities in LBE that are temporarily impossible to implement experiment. And interesting conclusions are disclosed which are stressed as follows:

Impurities saturation concentration distribution and solid particle migration are investigated in this work to better understand the CPs behaviors in the primary circuit of LBE reactor. The impurity concentrations of different element differ a lot which may help explain the formation of $\mathrm{Fe}-\mathrm{Cr}$ layer on the material surface and the element selective corrosion. When the particle diameter is greater than $100 \mu \mathrm{m}$, the driving force for the particle movement is buoyancy. The buoyancy will increase compared to other forces as the diameter augments. Consequently, the large particles tend to float on the free liquid surface or the solid-liquid interface.

As an outlook, a developed multi-phase model which combine the nucleation and dissolution, coagulation of solid impurities should be proposed to describe and predict more accurately and subtlety the impurity behaviors.

Supplementary Materials: The following are available online at www.mdpi.com/xxx/s1, Figure S1: title, Table S1: title, Video S1: title.

Author Contributions: Conceptualization, Ni.MY.; methodology, Wu.JW; software, Wang.YQ, He.JB; validation, Wu.RJ, Zeng.X. and Hu.C.; resources, Wu.RJ; writing-original draft preparation, Wu.JW.; writing-review and editing, Ni.MY;. All authors have read and agreed to the published version of the manuscript.

Funding: This research was funded by National Defense Pre-Research Foundation of China, grant No.201-XXXX0404-fzsdx-Tm06 and Fundamental Science on Nuclear Wastes and Environmental Safety Laboratory, grant No.18kfhk04

Acknowledgments: In this section, you can acknowledge any support given which is not covered by the author contribution or funding sections. This may include administrative and technical support, or donations in kind (e.g., materials used for experiments).

Conflicts of Interest: The authors declare no conflict of interest.

\section{References}

1. J. Zhang; N. Li. Review of the studies on fundamental issues in LBE corrosion. J. Nucl. Mater., vol. 373, no. 1-3, pp. 351-377, 2008, doi: 10.1016/j.jnucmat.2007.06.019.

2. R. G. Ballinger and J. Lim, "An overview of corrosion issues for the design and operation of high-temperature lead-and leadbismuth-cooled reactor systems," Nucl. Technol., vol. 147, no. 3, pp. 418-435, 2004.

3. B. F. Gromov, Y. I. Orlov, P. N. Martynov, and V. A. Gulevsky. The problems of technology of the heavy liquid metal coolants. Conference: Heavy liquid-metal coolants in the nuclear technologies, Obninsk, Russian Federation, 5-9 Oct 1998

4. Y. I. Orlov et al., "Hydrodynamic problems of heavy liquid metal coolants technology in loop-type and mono-block-type reactor installations," Nucl. Eng. Des., vol. 237, no. 15-17, pp. 1829-1837, 2007.

5. N. Li, "Active control of oxygen in molten lead--bismuth eutectic systems to prevent steel corrosion and coolant contamination," J. Nucl. Mater., vol. 300, no. 1, pp. 73-81, 2002.

6. G. Müller, G. Schumacher, and F. Zimmermann. Investigation on oxygen controlled liquid lead corrosion of surface treated steels. J. Nucl. Mater., vol. 278, no. 1, pp. 85-95, 2000.

7. J. Zhang, P. Hosemann, and S. Maloy. Models of liquid metal corrosion. J. Nucl. Mater., vol. 404, no. 1, pp. 82-96, 2010.

8. A. V Zrodnikov, A. D. Efanov, Y. I. Orlov, P. N. Martynov, V. M. Troyanov, and A. E. Rusanov. Heavy Liquid Metal Coolant-Lead--Bismuth and Lead-Technology. At. Energy, vol. 97, no. 2, pp. 534-537, 2004.

9. L. Koloszar. CFD simulation of the thermohydraulics of the MYRRHA reactor. Conference: THINS 2014 International Workshop. Modena, Italy. January 2014

10. D. Lilin et al. Multi-Physics Model Development for Polonium Transport Behavior in a Lead-Cooled Fast Reactor. Front. Energy Res., vol. 9, no. July, pp. 1-9, 2021, doi: 10.3389/fenrg.2021.711916.

11. L. Koloszar, S. Buckingham, P. Planquart, and S. Keijers. MyrrhaFoam: A CFD model for the study of the thermal hydraulic behavior of MYRRHA. Nucl. Eng. Des., vol. 312, pp. 256-265, 2017. 
12. A. Marino et al. Numerical modeling of iron-based corrosion product oxides mass transport in the MYRRHA reactor during normal operation. Nucl. Eng. Des., vol. 338, pp. 199-208, 2018.

13. A. Aerts, S. Gavrilov, G. Manfredi, A. Marino, K. Rosseel, and J. Lim. Oxygen--iron interaction in liquid lead--bismuth eutectic alloy. Phys. Chem. Chem. Phys., vol. 18, no. 29, pp. 19526-19530, 2016.

14. C. Fazio et al. Handbook on lead-bismuth eutectic alloy and lead properties, materials compatibility, thermal-hydraulics and technologies-2015 edition. 2015.

15. L. S. Crespo and others. Compatibility of structural materials with LBE and Pb: standardization of data, corrosion mechanism and rate. Handb. Lead--Bismuth Eutectic Alloy Lead Prop. Mater. Compat. Therm. Technol. Nucl. Energy Agency, Nucl. Sci. Comm., pp. 231-274, 2007.

16. J. Zhang and N. Li. Review of the studies on fundamental issues in LBE corrosion. J. Nucl. Mater., vol. 373, no. 1-3, pp. 351377, 2008.

17. Martynov P.N. Askhadullin R.Sh. Filtration purification of heavy liquid metal coolants from impurities. 2015. Problems of Atomic Science and Technology. Series: Nuclear and Reactor Constants.129-137

18. S. A. J. Morsi and A. J. Alexander. An investigation of particle trajectories in two-phase flow systems. J. Fluid Mech., vol. 55, no. 2, pp. 193-208, 1972. 